\title{
Freeze-in dark matter from a minimal $B-L$ model and possible grand unification
}

\author{
Rabindra N. Mohapatra ${ }^{1}$ and Nobuchika Okada $\odot^{2}$ \\ ${ }^{1}$ Maryland Center for Fundamental Physics and Department of Physics, University of Maryland, \\ College Park, Maryland 20742, USA \\ ${ }^{2}$ Department of Physics and Astronomy, University of Alabama, Tuscaloosa, Alabama 35487, USA
}

(Received 5 May 2020; accepted 5 June 2020; published 17 June 2020)

\begin{abstract}
We show that a minimal local $B-L$ symmetry extension of the standard model can provide a unified description of both neutrino mass and dark matter. In our model, $B-L$ breaking is responsible for neutrino masses via the seesaw mechanism, whereas the real part of the $B-L$ breaking Higgs field (called $\sigma$ here) plays the role of a freeze-in dark matter candidate for a wide parameter range. Since the $\sigma$ particle is unstable, for it to qualify as dark matter, its lifetime must be longer than $10^{25}$ seconds implying that the $B-L$ gauge coupling must be very small. This in turn implies that the dark matter relic density must arise from the freeze-in mechanism. The dark matter lifetime bound combined with dark matter relic density gives a lower bound on the $B-L$ gauge boson mass in terms of the dark matter mass. We point out parameter domains where the dark matter mass can be both in the $\mathrm{keV}$ to $\mathrm{MeV}$ range as well as in the PeV range. We discuss ways to test some parameter ranges of this scenario in collider experiments. Finally, we show that if instead of $B-L$, we consider the extra $U(1)$ generator to be $-4 I_{3 R}+3(B-L)$, the basic phenomenology remains unaltered and for certain gauge coupling ranges, the model can be embedded into a five-dimensional $S O(10)$ grand unified theory.
\end{abstract}

DOI: $10.1103 /$ PhysRevD.101.115022

\section{INTRODUCTION}

If small neutrino masses arise via the seesaw mechanism [1-5], the addition of a local $B-L$ symmetry [6,7] to the standard model (SM) provides a minimal scenario for beyond the standard model physics to achieve this goal. There are two possible classes of $B-L$ models: one where the $B-L$ generator contributes to the electric charge [6-8] and another where it does not [9-11]. In the first case, the $B-L$ gauge coupling $g_{B L}$ has a lower limit, whereas in the second case it does not and therefore can be arbitrarily small. There are constraints on the allowed ranges of $g_{B L}$ from different observations $[12,13]$ in the second case depending on whether there is or is not a dark matter particle in the theory. There are also possible ways to look for gravitational wave signals of $B-L$ breaking in the early Universe [14].

In Refs. [15,16], it was shown that if we added a $B-L$ charge carrying vectorlike fermion to the minimal $B-L$ model and want it to play the role of dark matter, new constraints emerge. In this note, we discuss an alternative

Published by the American Physical Society under the terms of the Creative Commons Attribution 4.0 International license. Further distribution of this work must maintain attribution to the author(s) and the published article's title, journal citation, and DOI. Funded by SCOAP ${ }^{3}$. possibility with the following new results. First is that the minimal version of the $B-L$ model itself, without any extra particles, can provide a dark matter (DM) candidate. The DM turns out to be the real part (denoted here as $\sigma$ ) of the complex $B-L=2$ Higgs field, that breaks $B-L$ and gives mass to the right-handed neutrinos in the seesaw formula. Even though this particle is not stable, there are certain allowed parameter ranges of the model, where its lifetime can be so long that it can play the role of a decaying dark matter. We isolate this parameter range and show that in this case, the freeze-in mechanism [17] can generate its relic density. We find this possibility to be interesting since it unifies both neutrino masses and dark matter in a single minimal framework [18]. We show how a portion of the parameter range of the model suggested by the dark matter possibility can be probed by the recently approved FASER experiment at the LHC [19] and other Lifetime Frontier experiments.

We then show that if we replace the $B-L$ symmetry by $\tilde{I} \equiv-4 I_{3 R}+3(B-L)$ (where $I_{3 R}$ is the right-handed weak isospin), the dark matter phenomenology remains largely unchanged and the model can be embedded into the $S O(10)$ grand unified theory in five space-time dimensions. Such a symmetry breaking of $S O(10)$ to $S U(5) \times U(1)_{\tilde{I}}$ has already been shown to arise from a symmetry breaking by a particular alignment for a vacuum expectation value (VEV) of a 45-dimensional Higgs field [20]. 
We note that there are examples of other models in the literature connecting neutrino mass generation mechanisms to dark matter; see, for example, Refs. [21,22].

This paper is organized as follows: in Sec. II, after briefly introducing the model, we discuss the lifetime of the $\sigma$ dark matter and its implications. In Sec. III, we discuss the small gauge coupling $g_{B L}$ range where the dark matter lifetime is long enough for it to play the role of dark matter. In Sec. IV, we show how freeze-in mechanism determines the relic density of dark matter and its implications for the allowed parameter range of the model. We also discuss how to test this model at the FASER and other Lifetime Frontier experiments. In Sec. V, we show that this model can also accommodate a PeV dark matter. In Sec. VI, we discuss the $S O$ (10) embedding of the closely allied model and in Sec. VII, we conclude with some comments and other implications of the model.

\section{BRIEF OVERVIEW OF THE MODEL}

Our model is based on the $U(1)_{B-L}$ extension of the SM with gauge quantum numbers under $U(1)_{B-L}$ determined by the baryon or lepton number of the particles. The gauge group of the model is $S U(3)_{c} \times S U(2)_{L} \times U(1)_{Y} \times U(1)_{B-L}$, where $Y$ is the SM hypercharge. We need three righthanded neutrinos (RHNs) with $B-L=-1$ to cancel the $B-L$ anomaly. The RHNs being SM singlets do not contribute to SM anomalies. The electric charge formula in this case is same as in the SM, i.e., $Q=I_{3 L}+\frac{Y}{2}$.

We break $B-L$ symmetry by giving a VEV to a $B-L=2 \mathrm{SM}$ neutral complex Higgs field $\Delta$, i.e., $\langle\Delta\rangle=$ $v_{B L} / \sqrt{2}$. This gives Majorana masses to the right-handed neutrinos $(N)$ via the coupling $f N N \Delta$. The real part of $\Delta$ (denoted by $\sigma$ ) is a physical field. Our goal in this paper is to show that $\sigma$ has the right properties to play the role of a dark matter of the universe. There are three challenges to achieving this goal which are as follows:

(i) The $\sigma$ field has couplings to the RHNs which in turn couple to SM particles providing a way for $\sigma$ to decay. Also, the $\sigma$ field has couplings to two $B-L$ gauge bosons $\left(Z_{B L}\right)$ which in turn couple to $\mathrm{SM}$ fields providing another channel for $\sigma$ to decay. In the next section, we show that there are parameter regions of the model where these decay modes give a long enough lifetimes for $\sigma$, so that it can be a viable unstable dark matter in the universe.

(ii) The second challenge is that for $\sigma$ to be a sole dark matter, it must account for the total observed relic density of the universe $\Omega_{\mathrm{DM}} h^{2} \simeq 0.12$ [23]. We show in Sec. IV that in the same parameter range, that gives rise to the long lifetime of $\sigma$, can also explain the observed relic density of dark matter via the freeze-in mechanism.

(iii) The $\sigma$ field could mix with the standard model Higgs field $h$ via the potential term $\lambda^{\prime} H^{\dagger} H \Delta^{\dagger} \Delta$ after symmetry breaking. However, it turns out that if we set $\lambda^{\prime}=0$ at the tree level, it can be induced at the one-loop level by fermion contributions and at the two-loop level from the top loop as shown in Ref. [11]. These induced couplings can be so small that they still lead to very long lifetimes for $\sigma$ in the parameter range of interest to us.

\section{DARK MATTER LIFETIME}

As noted earlier in Sec. II, the $\sigma$ field has couplings which could make it unstable and thereby disqualify it from being a dark matter. However, we will show that there is a viable parameter range of the model where this decay lifetime is longer than $10^{25}$ seconds [24] so that it can be a dark matter candidate. We discuss the following two modes now:

(i) Decay mode $\sigma \rightarrow N N \rightarrow \ell f \bar{f} \ell f \bar{f}$ : The decay width for this process is estimated as

$$
\Gamma_{N N} \simeq \frac{\left(f h_{\nu}^{2} h_{\mathrm{SM}}^{2}\right)^{2}}{(4 \pi)^{8}} \frac{m_{\sigma}^{13}}{M_{N}^{4} m_{h}^{8}},
$$

where $h_{\nu}$ is a neutrino Dirac Yukawa coupling, $h_{\mathrm{SM}}$ is a Yukawa coupling of an SM fermion $f$, and $m_{h}=$ $125 \mathrm{GeV}$ is the SM Higgs boson mass. For a GeV mass $\sigma$ and $\mathrm{TeV}$ mass $\mathrm{RHN}$, the lifetime of $\sigma$ turns out to be $\tau_{\sigma}[\mathrm{sec}] \sim 10^{37} /\left(f^{2} h_{\mathrm{SM}}^{4}\right)$, which is quite consistent with the requirement for it to be a dark matter. Here, we have used the seesaw formula $h_{\nu}^{2} v_{\mathrm{EW}}^{2} / M_{N} \simeq m_{\nu}$ with $v_{\mathrm{EW}}=246 \mathrm{GeV}$ and $\mathrm{a}$ typical neutrino mass scale $m_{\nu} \simeq 0.1 \mathrm{eV}$.

(ii) Decay mode $\sigma \rightarrow Z_{B L} Z_{B L} \rightarrow f \bar{f} f \bar{f}$ : The decay width for this process is

$\Gamma_{Z_{B L} Z_{B L}} \simeq \frac{\left(2 g_{B L}\right)^{4} v_{B L}^{2} g_{B L}^{4} m_{\sigma}^{7}}{(4 \pi)^{5} M_{Z_{B L}}^{8}}=\frac{g_{B L}^{6}}{256 \pi^{5}} \frac{m_{\sigma}^{7}}{M_{Z_{B L}}^{6}}$.

This mode is sensitive to the values of $g_{B L}$ as well as $M_{Z_{B L}}$. The estimate of $\tau_{\sigma}$ due to this decay mode is given by

$$
\begin{aligned}
\tau_{\sigma} \simeq & 5.2 \times 10^{-20}\left(\frac{1}{g_{B L}}\right)^{6}\left(\frac{1 \mathrm{GeV}}{m_{\sigma}}\right)^{7} \\
& \times\left(\frac{M_{Z_{B L}}}{1 \mathrm{GeV}}\right)^{6} \text { seconds. }
\end{aligned}
$$

Imposing $\tau_{\sigma}>10^{25}$ seconds, this puts an upper bound on the $g_{B L}$ as a function of $M_{Z_{B L}}$ and $m_{\sigma}$,

$$
g_{B L} \leq 4.2 \times 10^{-8}\left(\frac{M_{Z_{B L}}}{1 \mathrm{GeV}}\right)\left(\frac{1 \mathrm{GeV}}{m_{\sigma}}\right)^{7 / 6} .
$$

We find that the allowed regions where the $\sigma$ field can be a dark matter correspond to a very small $g_{B L}$ coupling. For instance, for $m_{\sigma} \sim 1 \mathrm{GeV}$ and $M_{Z_{B L}} \sim$ $1 \mathrm{TeV}$, we find that $g_{B L} \lesssim 4 \times 10^{-5}$. 

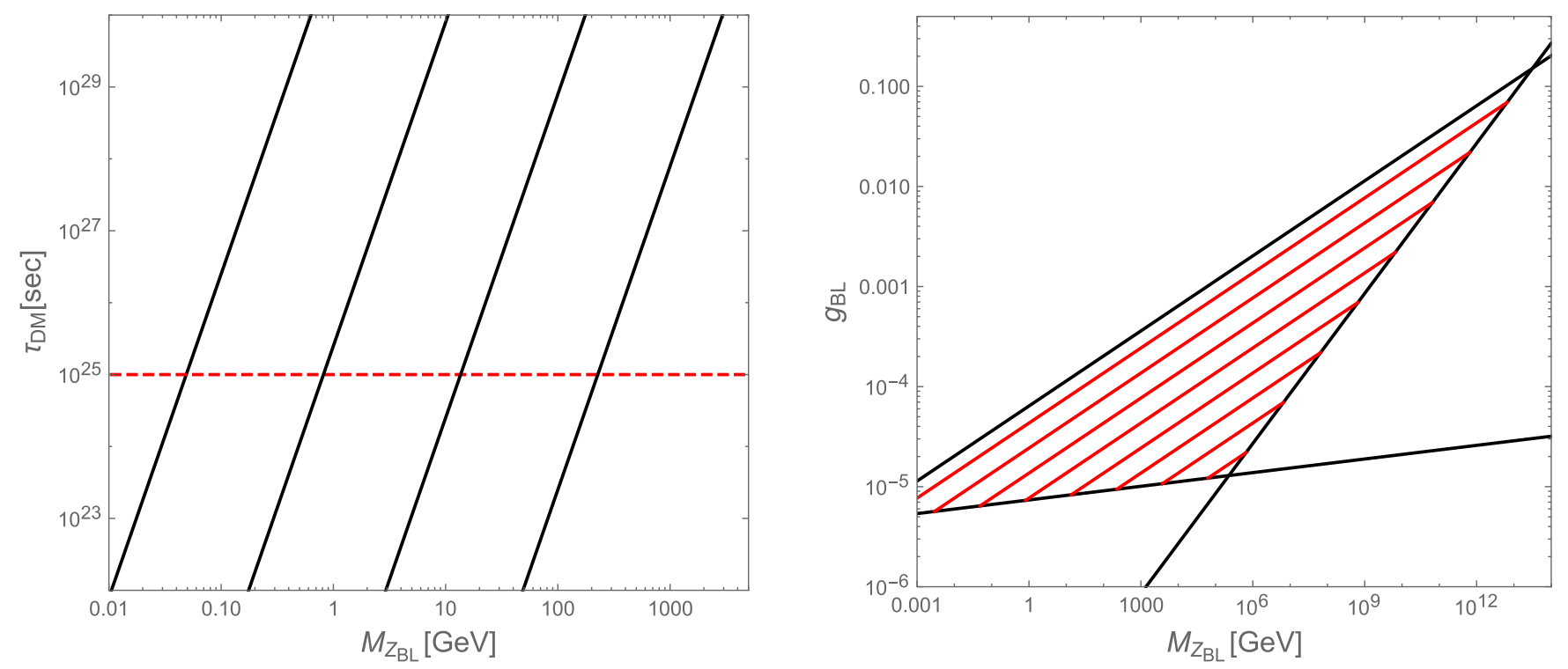

FIG. 1. Left panel: the dark matter $\sigma$ lifetime as a function of $M_{Z_{B L}}$. The diagonal solid lines correspond to $m_{\sigma}=1 \mathrm{MeV}, 10 \mathrm{MeV}$, $100 \mathrm{MeV}$, and $1 \mathrm{GeV}$ from left to right, along which the observed DM relic density of $\Omega_{\mathrm{DM}} h^{2}=0.12$ is reproduced. Right panel: the $g_{B L}$ values as a function of $M_{Z_{B L}}$ from the requirement of relic density buildup. Different red lines correspond to different DM masses $\left(m_{\sigma}\right.$ starting with $10 \mathrm{keV}$ at the top and as we go below, we go in steps of a factor of 10 to $100 \mathrm{keV}, 1 \mathrm{MeV}$, etc., till $100 \mathrm{GeV}$ ) that satisfy the relic density constraint, i.e., $\Omega_{\mathrm{DM}} h^{2}=0.12$. Two diagonal black lines denote the condition of Eq. (6), and the horizontal black line corresponds to Eq. (14).

(iii) We now comment on the $\sigma$-Higgs mixing effect on the DM lifetime. To keep the lifetime above limit $\tau_{\sigma}>10^{25}$ seconds, we set the tree-level $H-\Delta$ coupling in the Higgs potential to zero so that $\sigma$ and the SM Higgs field $h$ do not mix at the tree level. This will, for example, be true if the model becomes supersymmetric at a high scale. The $\sigma$-Higgs mixing in this case is loop induced as shown in Ref. [11] and for the parameter range of interest to us, can be small enough to satisfy the DM lifetime constraint as we show below.

For the case when $m_{\sigma} \leq m_{h}$, the dominant contribution to the loop induced mixing comes from an RHN fermion box diagram. This contribution is logarithmically divergent. Using the Planck mass as the cutoff, we can estimate the mixing angle to be $\theta \sim \frac{f^{2} h_{\nu}^{2}}{16 \pi^{2}} \frac{v_{\mathrm{EW}} v_{B L}}{m_{h}^{2}} \sim \frac{1}{16 \pi^{2}} \frac{m_{\nu} M_{N}^{3}}{v_{\mathrm{EW}} m_{h}^{2}} \frac{2 g_{B L}}{M_{B L}}$. Through this mixing, the DM particle can decay to a pair of SM fermions with a partial decay width of $\Gamma_{\sigma \rightarrow f \bar{f}} \sim \frac{\theta^{2}}{4 \pi}\left(\frac{m_{f}}{v_{\mathrm{EW}}}\right)^{2} m_{\sigma}$. The lifetime constraint then translates to a limit on $g_{B L}$ as follows:

$g_{B L}<2.8 \times 10^{-6}\left(\frac{v_{\mathrm{EW}}}{m_{f}}\right)\left(\frac{1 \mathrm{GeV}}{m_{\sigma}}\right)^{1 / 2}\left(\frac{1 \mathrm{GeV}}{M_{N}}\right)^{3}\left(\frac{M_{Z_{B L}}}{1 \mathrm{GeV}}\right)$.

With a suitable choice of $M_{N}\left(>m_{\sigma}\right)$, we can see that this limit is quite compatible with our results shown in the right panel of Figs. 1 and 2.
For the case when $m_{\sigma}>m_{h}$, on the other hand, the DM particle can decay to a pair of Higgs doublets through the mixing, and we find that the loop induced mixing is not

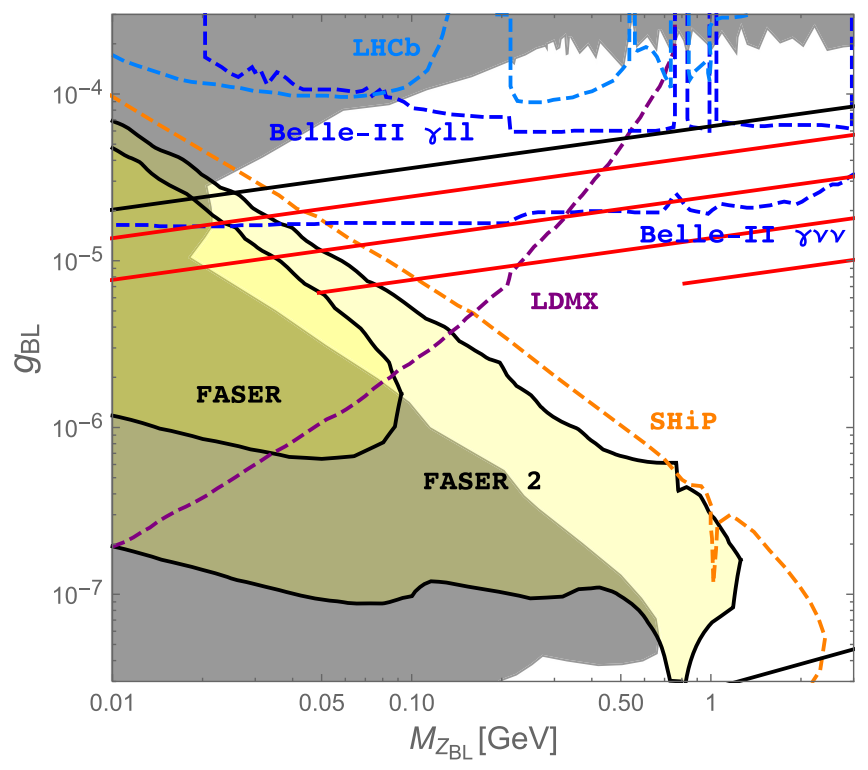

FIG. 2. FASER reachable region of the parameter space of our model. The black lines at the top and bottom denote the upper and lower limits on the $g_{B L}$ [Eq. (6)]. The red lines correspond to $m_{\sigma}=10 \mathrm{keV}, 100 \mathrm{keV}, 1 \mathrm{MeV}$, and $10 \mathrm{MeV}$ from top to bottom, respectively, along which $\Omega_{\mathrm{DM}}=0.12$ is satisfied. The parameter region of $10 \mathrm{keV} \lesssim m_{\sigma} \lesssim 1 \mathrm{MeV}$ and $10 \mathrm{MeV} \lesssim M_{Z_{B L}} \lesssim$ a few $\mathrm{GeV}$ can be tested by various Lifetime Frontier experiments in the near future. 

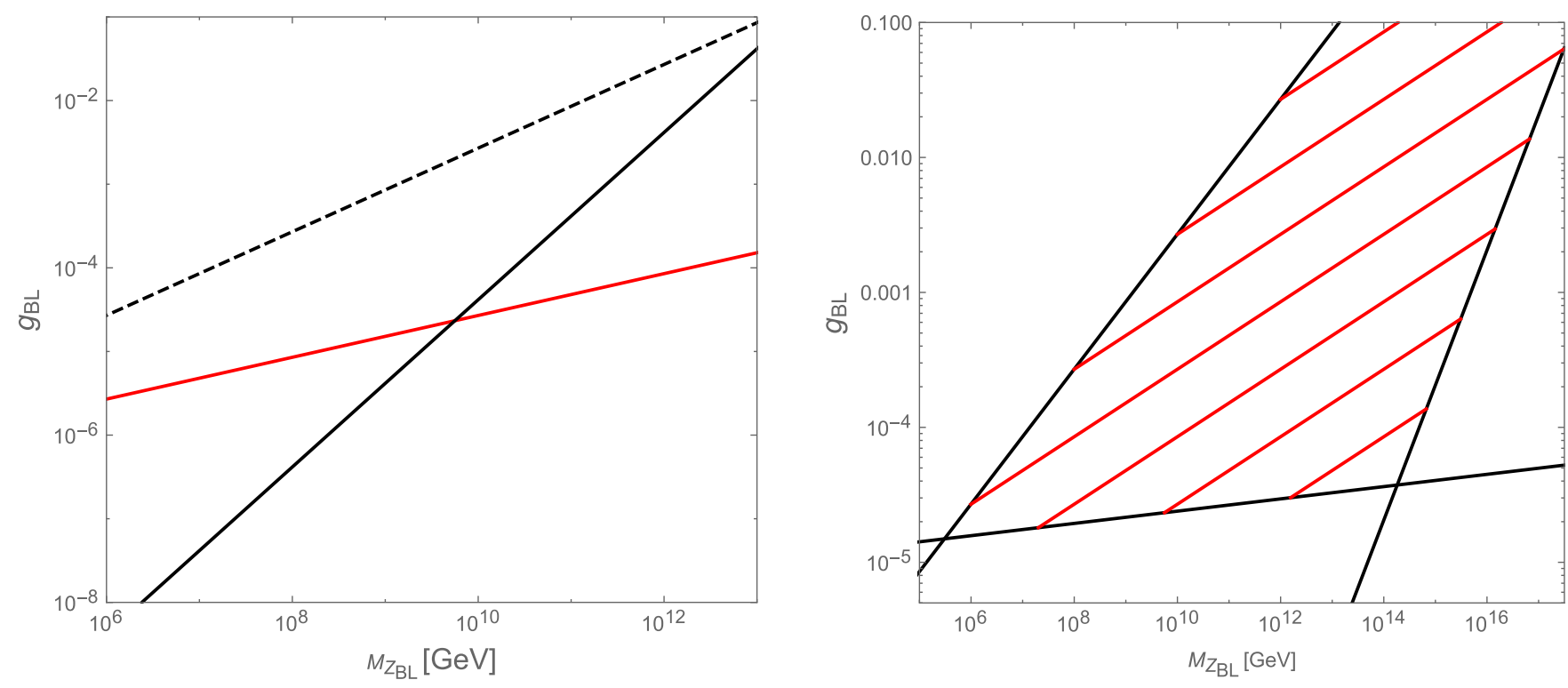

FIG. 3. Left panel: the red line corresponds to DM mass $m_{\sigma}=1 \mathrm{PeV}$ with $\Omega_{\mathrm{DM}} h^{2}=0.12$. This corresponds to the case where the DM is produced by $f \bar{f} \rightarrow Z_{B L} \sigma$. The left of dashed line corresponds to the DM being in thermal equilibrium and therefore is not the area for freeze-in case. The left of black solid line corresponds to $\tau_{\sigma}<10^{25}$ seconds and is excluded. Right panel: the red lines represent the DM masses from top $100 \mathrm{keV}, 10 \mathrm{MeV}, 1 \mathrm{GeV}$ (jump of 100 times) till $100 \mathrm{PeV}$ being the lowest red line. Along the red line $\Omega_{\mathrm{DM}} h^{2}=0.12$ is satisfied. The lower black line comes from Eq. (21). The upper black line corresponds to $Z_{B L}$ not being in equilibrium. The condition of $v_{B L} \leq M_{P}$ is depicted by the right diagonal black line.

small enough to be consistent with the results shown in the right panels of Figs. 1 and 3. In this case, we consider a cancellation of the mixing between the tree- and loop-levels contributions.

We will now explore whether for such small parametric values for $g_{B L}$, we can generate the observed dark matter relic density of the universe.

\section{RELIC DENSITY}

\section{A. Allowed range of $\boldsymbol{g}_{\boldsymbol{B} L}$ from preconditions to freeze-in}

The first point to notice is that for $\mathrm{GeV}$ scale DM $(\sigma)$, for values of $g_{B L}$ that satisfy the lifetime constraint, the $\sigma$ field is out of equilibrium from the SM particles. Therefore, the standard thermal freeze-out mechanism for creation of DM relic density does not apply and one has to explore the freeze-in mechanism. For this to work, we need the $Z_{B L}$ field, whose annihilation will produce the DM, to be in equilibrium with the SM fields. This question was explored in Ref. [15] and it was pointed out that the most efficient process for $Z_{B L}$ to be in equilibrium with SM particles is via the process $f \bar{f} \rightarrow Z_{B L}+\gamma$. The condition on $g_{B L}$ for this to happen is $g_{B L}>2.7 \times 10^{-8}\left(\frac{M_{Z_{B L}}}{1 \mathrm{GeV}}\right)^{1 / 2}$.

An upper bound on $g_{B L}$ comes from the fact that the DM particle $\sigma$ is out of equilibrium in the early universe. The first process to consider is $Z_{B L} Z_{B L} \leftrightarrow \sigma \sigma$ for which the out-of-equilibrium condition is given by $n_{\sigma}\langle\sigma v\rangle<H$. Here $n_{\sigma} \sim T^{3}$ is the number density of the $\mathrm{DM} \sigma,\langle\sigma v\rangle \sim g_{B L}^{4} /\left(4 \pi T^{2}\right)$, and the Hubble parameter $H=\sqrt{\frac{\pi^{2}}{90} g_{*}} T^{2} / M_{P}$ with the reduced Planck mass
$M_{P}=2.43 \times 10^{18} \mathrm{GeV}$ and the effective total number of relativistic degrees of freedom $g_{*}$ (we set $g_{*}=106.75$ for the SM particle plasma in our analysis throughout this paper). Requiring that this inequality is satisfied until $T \sim M_{Z_{B L}}$, we find that $g_{B L}<6.4 \times 10^{-5}\left(\frac{M_{Z_{B L}}}{1 \mathrm{GeV}}\right)^{1 / 4}$. Combining with the equilibrium condition for $Z_{B L}$, we find that we have to work in the range of $g_{B L}$ values

$2.7 \times 10^{-8}\left(\frac{M_{Z_{B L}}}{1 \mathrm{GeV}}\right)^{1 / 2}<g_{B L}<6.4 \times 10^{-5}\left(\frac{M_{Z_{B L}}}{1 \mathrm{GeV}}\right)^{1 / 4}$

to generate the relic density.

There is another upper bound on $g_{B L}$ that arises from the fact that the process $N N \rightarrow \sigma \sigma$ should also out of equilibrium. The reason is that in the early universe, the righthanded neutrinos are always in equilibrium with SM particles via processes such as $N+t \leftrightarrow \nu+t$ etc., and $N \leftrightarrow H \ell$ for $M_{N}>m_{h}$. If $N N \leftrightarrow \sigma \sigma$ is also in equilibrium, the freeze-in mechanism for relic density generation of $\sigma$ will not work. To get this upper bound on $g_{B L}$ using this condition, we use $n_{\sigma}\left\langle\sigma_{N N \rightarrow \sigma \sigma} v\right\rangle<H$ at $T \sim M_{N}$ and find

$$
\frac{1}{4 \pi}\left(\frac{M_{N}^{5}}{v_{B L}^{4}}\right)<\sqrt{\frac{\pi^{2}}{90} g_{*}} \frac{M_{N}^{2}}{M_{P}} .
$$

Using $M_{Z_{B L}}=2 g_{B L} v_{B L}$, this leads to 


$$
g_{B L}<3.2 \times 10^{-5}\left(\frac{M_{Z_{B L}}}{1 \mathrm{GeV}}\right)^{1 / 4}\left(\frac{M_{Z_{B L}}}{M_{N}}\right)^{3 / 4}
$$

Note that for $M_{N} \sim M_{Z_{B L}}$, this upper limit is about the same level as in Eq. (6) so that indeed the freeze-in mechanism is called for in creating the relic density buildup. In the following, we consider $M_{N}<M_{Z_{B L}}$, for which the upper bound is determined by the $B-L$ gauge interaction. Incidentally, we note that if $M_{N}<m_{h}$, the interactions of the RHNs with the SM particles are too week for them to be in thermal equilibrium, and the above discussion is not applicable. ${ }^{1}$

\section{B. Relic density buildup}

In order to calculate the relic density buildup via the freeze-in mechanism, we solve the following Boltzmann equation (defining $x=\frac{m_{\sigma}}{T}$ ):

$$
\frac{d Y}{d x} \simeq \frac{\langle\sigma v\rangle}{x^{2}} \frac{s\left(m_{\sigma}\right)}{H\left(m_{\sigma}\right)} Y_{e q}^{2}
$$

where $Y$ is the yield of the DM $\sigma, Y_{e q}$ is $Y$ if the DM $\sigma$ is in thermal equilibrium, and $s\left(m_{\sigma}\right)$ and $H\left(m_{\sigma}\right)$ are the entropy density and the Hubble parameter, respectively, evaluated at $T=m_{\sigma}$. For the DM particle creation process $Z_{B L} Z_{B} \rightarrow \sigma \sigma$, we approximate $\langle\sigma v\rangle \simeq \frac{g_{B L}^{4}}{4 \pi T^{2}}=\frac{g_{B L}^{4}}{4 \pi} \frac{x^{2}}{m_{\sigma}^{2}}$. Note that this formula is applicable for $T \geq M_{Z_{B L}} \gg m_{\sigma}$. The reason for this is that for $T \leq M_{Z_{B L}}$, the number density of $Z_{B L}$ is Boltzmann suppressed and $\sigma$ particle creation stops. Using $\frac{S\left(m_{\sigma}\right)}{H\left(m_{\sigma}\right)} \simeq 14 m_{\sigma} M_{P}$ and $Y_{e q} \simeq 2.2 \times 10^{-3}$ and integrating the above equation from $x_{R H}$ to $x$ (where $x_{R H}=$ $m_{\sigma} / T_{R H}$ with the reheating temperature after inflation $\left.T_{R H} \gg M_{Z_{B L}}\right)$, we obtain

$Y(x)-Y\left(x_{R H}\right) \simeq 5.1 \times 10^{-6} g_{B L}^{4}\left(\frac{M_{P}}{m_{\sigma}}\right)\left(x-x_{R H}\right)$.

Then taking $Y(\infty) \simeq Y\left(x_{B L}=m_{\sigma} / M_{Z_{B L}}\right)$, we estimate the DM relic density,

$\Omega_{\mathrm{DM}} h^{2} \simeq \frac{m_{\sigma} s_{0} Y(\infty)}{\rho_{0} / h^{2}} \simeq 3.4 \times 10^{21} g_{B L}^{4}\left(\frac{m_{\sigma}}{1 \mathrm{GeV}}\right)\left(\frac{1 \mathrm{GeV}}{M_{Z_{B L}}}\right)$,

where $s_{0}=2890 / \mathrm{cm}^{3}$ is the entropy density of the present universe, and $\rho_{c} / h^{2}=1.05 \times 10^{-5} \mathrm{GeV} \mathrm{cm}^{3}$ is the critical density. This leads to the following expression for $g_{B L}$ :

\footnotetext{
${ }^{1}$ Note also that, as a general possibility, if $M_{N}$ is greater than the reheating temperature after inflation $\left(T_{R H}\right)$, then RHN is irrelevant to our DM physics discussion.
}

$$
g_{B L} \simeq 2.4 \times 10^{-6}\left(\frac{M_{Z_{B L}}}{1 \mathrm{GeV}}\right)^{1 / 4}\left(\frac{1 \mathrm{GeV}}{m_{\sigma}}\right)^{1 / 4}
$$

to reproduce the observed DM relic density $\Omega_{\mathrm{DM}} h^{2}=0.12$.

Using Eq. (12) in Eq. (3), we show the lifetime for various values of $m_{\sigma}$ in Fig. 1 (left panel). The diagonal lines from left to right correspond to $m_{\sigma}=1 \mathrm{MeV}$, $10 \mathrm{MeV}, 100 \mathrm{MeV}$, and $1 \mathrm{GeV}$, respectively, along which $\Omega_{\mathrm{DM}} h^{2}=0.12$ is reproduced. The horizontal dashed line indicates the astrophysical bound on $\tau_{\sigma}>10^{25}$ seconds. Combining Eqs. (4) and (12), we obtain a lower bound on $M_{Z_{B L}}$,

$$
M_{Z_{B L}} \gtrsim 210\left(\frac{m_{\sigma}}{1 \mathrm{GeV}}\right)^{11 / 9} \mathrm{GeV}
$$

In the same way but eliminating $m_{\sigma}$, we find a lower bound on $g_{B L}$,

$$
g_{B L} \gtrsim 7.2 \times 10^{-6}\left(\frac{M_{Z_{B L}}}{\mathrm{GeV}}\right)^{1 / 22}
$$

Considering all the constraints from Eqs. (6), (12), and (14), we show the allowed parameter region in Fig. 1 (right panel). The region between two diagonal black lines satisfies the condition of Eq. (6) and the horizontal black line corresponds to Eq. (14). The observed $\Omega_{\mathrm{DM}} h^{2}=0.12$ is reproduced along the red lines each of which corresponds to a fixed $m_{\sigma}$ value. In the right panel, the region for $M_{Z_{B L}} \lesssim 10 \mathrm{MeV}$ and $g_{B L} \sim 10^{-5}$ is excluded by the longlived $Z_{B L}$ boson search results. See Fig. 2 for details.

In the right panel of Fig. 1, we can see that there is an allowed parameter region for $g_{B L}=\mathcal{O}\left(10^{-5}\right)$ and $M_{Z_{B L}}=$ $1 \mathrm{MeV}-1 \mathrm{GeV}$. For the parameter region, $Z_{B L}$ boson can be long-lived and such a long-lived neutral particle can be explored in the near future by the Lifetime Frontier experiments, such as FASER [19], SHiP [25], LDMX [26], Belle II [27], and LHCb [28,29]. The $Z_{B L}$ boson search of the FASER experiment at the LHC is summarized in Ref. [19] along with the search reaches of other experiments as well as the current excluded region [30]. In Fig. 2, we show our results of the right panel of Fig. 2 along with the summary plot in Ref. [19]. The red lines correspond to $m_{\sigma}=10 \mathrm{keV}, 100 \mathrm{keV}, 1 \mathrm{MeV}$, and $10 \mathrm{MeV}$ from top to bottom, respectively. The parameter region of $10 \mathrm{keV} \lesssim m_{\sigma} \lesssim 1 \mathrm{MeV}$ and $10 \mathrm{MeV} \lesssim M_{Z_{B L}} \lesssim$ a few $\mathrm{GeV}$ can be tested by various Lifetime Frontier experiments in the near future.

Before moving on to the next section, we comment on the dark matter production processes involving the RHN. If the RHN is in thermal equilibrium, the DM particles can also be created through $N N \rightarrow \sigma \sigma$. The estimate of $Y(\infty)$ from this process is analogous to the process $Z_{B L} Z_{B L} \rightarrow \sigma \sigma$, and resultant density is roughly given by Eq. (11) with replacing $g_{B L} \rightarrow f$ and $M_{Z_{B L}} \rightarrow M_{N}$. Thus, 
we take $M_{N}<M_{Z_{B L}}$, or equivalently $f<g_{B L}$, so that the RHN mediated DM production becomes subdominant. Calculations for other processes such as $N N \rightarrow Z_{B L} \sigma$ and $N Z_{B L} \rightarrow N \sigma$ are also analogous, and we can arrive at the same conclusion. We can also consider DM production processes through Dirac Yukawa couplings $\left(h_{\mathrm{SM}}\right)$ such as $N H \rightarrow \ell \sigma$ and $H \ell \rightarrow N \sigma$, where $H$ and $\ell$ are the Higgs and lepton doublets, respectively. The DM productions can be subdominant if $h_{\mathrm{SM}}$ is sufficiently small; in other words, through the seesaw formula, $N$ is sufficiently light. The discussion for the DM production process of $H \ell \rightarrow N \sigma$ is applicable even if the RHN is not in thermal equilibrium.

\section{PEV DARK MATTER FROM $B-L$ BREAKING}

So far, we have explored the lower mass range of the dark matter. In this section, we explore the possibility that the $\sigma$ mass is in the $\mathrm{PeV}$ range so that one could attempt to explain the $100 \mathrm{TeV}$ to $\mathrm{PeV}$ neutrinos observed in IceCube Neutrino Observatory [31] by using $\sigma$ decay. We do not attempt to explain the IceCube signal here but simply to raise the possibility that a $\mathrm{PeV}$ mass $\sigma$ can also qualify as the dark matter in our model in a different parameter range. For this purpose, let us go through all the constraints on the model discussed above for this case.

\section{A. Lifetime constraint}

This constraint is same as in the case of light $\sigma$ in Eq. (4) except that in the right-hand side, the masses of $\sigma$ and $Z_{B L}$ are now higher and the new constraint can be written as

$$
g_{B L} \leq 4.2 \times 10^{-9}\left(\frac{M_{Z_{B L}}}{1 \mathrm{PeV}}\right)\left(\frac{1 \mathrm{PeV}}{m_{\sigma}}\right)^{7 / 6} .
$$

If we restrict the $B-L$ breaking VEV $v_{B L} \leq 10^{16} \mathrm{GeV}$, then the lifetime constraint can be translated to $M_{Z_{B L}} \sim$ $10^{10} \mathrm{GeV}$ for $g_{B L}$ as large as $10^{-5}$.

We note that the one-loop $\sigma-h$ mixing contribution in this case leads to a very strong upper limit on the $g_{B L}$ value and much too small to generate enough relic density for the dark matter. In this case, therefore, we fine-tune the treelevel and one-loop $\sigma$-Higgs coupling to zero.

\section{B. Relic density constraints}

We next explore the constraints of relic density on the heavy DM case. For such low $g_{B L}$ values, a heavy $\mathrm{PeV}$ scale DM and the $10^{10} \mathrm{GeV}$ or higher mass $Z_{B L}$ would never have been in equilibrium. The relic density must arise as in the first case via the freeze-in mechanism. Since $Z_{B L}$ is not in thermal equilibrium, the production takes place via the process $f \bar{f} \rightarrow Z_{B L} \sigma$ through the SM fermion pair annihilations in the thermal plasma. In this case, the Boltzmann equation is given by

$$
\frac{d Y}{d x} \simeq \frac{\langle\sigma v\rangle}{x^{2}} \frac{s\left(m_{\sigma}\right)}{H\left(m_{\sigma}\right)} Y_{e q} Y_{e q}^{B L},
$$

where $Y_{e q}^{B L}$ is the yield of $Z_{B L}$ in thermal equilibrium and the cross section for the process $f \bar{f} \rightarrow Z_{B L} \sigma$ is estimated as

$$
\langle\sigma v\rangle=\frac{g_{B L}^{4}}{4 \pi} \frac{M_{Z_{B L}}^{2}}{m_{\sigma}^{4}} x^{4} .
$$

Recall that the DM production stops at $T \simeq M_{Z_{B L}}$ due to kinematics. Using $Y_{e q}^{B L} \simeq 2 Y_{e q}$ for $T \gtrsim M_{Z_{B L}}^{2} \gg m_{\sigma}$, we integrate the Boltzmann equation from $x_{R H}$ to $x_{B L}=\frac{m_{\sigma}}{M_{Z_{B L}}}$ and obtain

$$
\begin{aligned}
Y\left(x_{B L}\right) & \simeq 3.4 \times 10^{-6} g_{B L}^{4}\left(\frac{M_{Z_{B L}}}{m_{\sigma}}\right)^{2}\left(\frac{M_{P}}{m_{\sigma}}\right)\left(x_{B L}^{3}-x_{R H}^{3}\right) \\
& \simeq 3.4 \times 10^{-6} g_{B L}^{4}\left(\frac{M_{P}}{M_{Z_{B L}}}\right),
\end{aligned}
$$

where we have used $Y\left(x_{R H}\right)=0$ and $x_{R H} \gg x_{B L}$. We now use, as before, $Y(\infty) \simeq Y\left(x_{B L}\right)$ and estimate the DM relic density,

$\Omega_{\mathrm{DM}} h^{2} \simeq \frac{m_{\sigma} s_{0} Y(\infty)}{\rho_{0} / h^{2}} \simeq 2.3 \times 10^{21} g_{B L}^{4}\left(\frac{m_{\sigma}}{1 \mathrm{GeV}}\right)\left(\frac{1 \mathrm{GeV}}{M_{Z_{B L}}}\right)$.

In order to reproduce $\Omega_{\mathrm{DM}} h^{2}=0.12$, we find

$$
g_{B L} \simeq 2.7 \times 10^{-6}\left(\frac{M_{Z_{B L}}}{m_{\sigma}}\right)^{1 / 4} .
$$

Combining Eqs. (15) and (20), we find

$$
g_{B L} \gtrsim 1.6 \times 10^{-5}\left(\frac{M_{Z_{B L}}}{1 \mathrm{PeV}}\right)^{1 / 22} .
$$

We require that the $Z_{B L}$ is not in equilibrium which gives the consistency condition

$$
g_{B L}<2.7 \times 10^{-8}\left(\frac{M_{Z_{B L}}}{\mathrm{GeV}}\right)^{1 / 2} .
$$

In Fig. 3 (left panel), we show our result for $m_{\sigma}=1 \mathrm{PeV}$. The dashed line denotes the upper bound on $g_{B L}$ from the out-of-equilibrium condition of Eq. (22). The diagonal black line shows the lifetime constraint of Eq. (4), or equivalently Eq. (15). Along the red line, the observed DM relic density is reproduced [see Eq. (20)]. In the figure, we find the lower bound on $M_{Z_{B L}}=$ $4.5 \times 10^{9} \mathrm{GeV}$. In the right panel of Fig. 3, we show the results for various values of $m_{\sigma}$. The red lines from top to bottom correspond to the results for $m_{\sigma}=100 \mathrm{keV}$, 
$10 \mathrm{MeV}, 1 \mathrm{GeV}, 100 \mathrm{GeV}, 10 \mathrm{TeV}, 1 \mathrm{PeV}$, and $100 \mathrm{PeV}$, respectively. The left diagonal black line denotes the outof-equilibrium condition of Eq. (22), while the horizontal black line corresponds to Eq. (21). We also impose a condition of $v_{B L} \leq M_{P}$, which is depicted by the right diagonal black line. We thus see that there is enough parameter range in the model for the dark matter to be in the $\mathrm{PeV}$ range so that it can be relevant to the $\mathrm{PeV}$ neutrinos observed in IceCube experiment. This is possible for $M_{Z_{B L}} \gtrsim 10^{10} \mathrm{GeV}$ and $v_{B L} \gtrsim 10^{14} \mathrm{GeV}$.

\section{PROSPECTS FOR SO(10) EMBEDDING}

In this section, we like to point out that a slight variation of the model leads to its possible embedding into $S O(10)$ grand unified theory (GUT), which we believe should add to its theoretical appeal as a minimal GUT model that unifies neutrino masses and dark matter. The starting point of this discussion is the observation that the hypercharge generator $Y$ is a linear combination of the $I_{3 R}$ and the normalized $B-L$ generators $I_{B L}$ of $S O(10)$ as follows:

$$
Y=I_{3 R}+\sqrt{\frac{2}{3}} I_{B L}
$$

where $I_{B L}=\sqrt{\frac{3}{2}} \frac{B-L}{2}$. The $B-L$ generator in the main body of the paper is not orthogonal to the $Y$ generator defined above. Therefore, it cannot emerge from $S O(10)$ breaking since $I_{B L}$ is not orthogonal to $Y$ defined above. Instead, if we consider the generator $\tilde{I} \equiv-4 I_{3 R}+$ $3(B-L)$, we get $\operatorname{Tr}(\tilde{I} Y)=0$ (i.e., they are orthogonal) for any irreducible representation of $S O(10)$ and can therefore emerge from $S O(10)$ breaking. This generator was also identified in Ref. [32] as the generator $U(1)_{X}$ for $x_{H}=-4 / 5$. Indeed, it has been shown in Ref. [20] that such a generator emerges out of $S O(10)$ breaking by a $\mathbf{4 5}$ Higgs field. To see this note that 45 Higgs under $S U(3) \times$ $S U(2)_{L} \times S U(2)_{R} \times U(1)_{B-L}$ group has multiplets $(1,1,1,0)$ and $(1,1,3,0)$ which can take VEVs $\omega_{Y}$ and $\omega_{B L}$, respectively. If we fine-tune the parameters of the Higgs potential, we can get $\omega_{Y}=\omega_{B L}$ in which case the unbroken generators are $U(1)_{Y} \times U(1)_{\tilde{I}}$. The normalized $\tilde{I}=\frac{1}{2 \sqrt{10}}\left(-4 I_{3 R}+3(B-L)\right)$.

As it turns out, the dark matter phenomenology discussed above remains unchanged if we use the Higgs field $\sigma$ to break the $U(1)_{\tilde{I}}$ symmetry. The $\sigma$ field then emerges from the 126-dimensional representation of $S O(10)$ and our dark matter field $\sigma$ has $\tilde{I}=\frac{\sqrt{10}}{2}$ and therefore has all the properties required above for our dark matter. Again, as before, we fine-tune parameters to get the desired dark matter $(\sigma)$. Our goal here is not to construct a natural model but rather to see whether it is phenomenologically possible to have $\sigma$ play the role of dark matter.
Our scenario for $S O(10)$ breaking is as follows: we use 45-dimensional Higgs field to break $S O(10)$ down to $S U(5) \times U(1)_{\tilde{I}}$ by choosing the vacuum with $\omega_{Y}=\omega_{B L}$, as noted above. The $\tilde{I}$ quantum numbers of fermions are then given by $\tilde{I}(\mathbf{1 0})=\frac{1}{2 \sqrt{10}}, \tilde{I}(\overline{\mathbf{5}})=\frac{-3}{2 \sqrt{10}}$ and $\tilde{I}(\mathbf{1})=\frac{5}{2 \sqrt{10}}$, where $\mathbf{1 0}, \overline{\mathbf{5}}$, and $\mathbf{1}$ are the $S U(5)$ representations in $S O(10)$ spinor 16. For a 10-representation Higgs field in $S O(10)$, which is decomposed into $\mathbf{5}+\overline{\mathbf{5}}$ under $S U(5)$ and includes the SM Higgs doublet, the $\tilde{I}$ quantum numbers are given by $\tilde{I}(\mathbf{5})=\frac{-2}{2 \sqrt{10}}$ and $\tilde{I}(\mathbf{5})=\frac{2}{2 \sqrt{10}}$.

Let us now discuss the evolution of the $\tilde{I}$ gauge coupling. The evolution of $U(1)_{\tilde{I}}$ gauge coupling $\left(g_{\tilde{I}}\right)$ is given by

$$
\mu \frac{d \alpha_{\tilde{I}}^{-1}}{d \mu}=\frac{b_{\tilde{I}}}{2 \pi},
$$

where $b_{I}=-49 / 10$ at a scale $\mu$ below the $S U(5)$ unification while $b_{\tilde{I}}=-5$ in $S U(5) \times U(1)_{\tilde{I}}$ theory by considering that the SM Higgs doublet is embedded into a 5-representation in $S U(5)$. For simplicity, we have assumed that in each step of the gauge symmetry breaking, $S O(10) \rightarrow S U(5) \times U(1)_{\tilde{I}} \rightarrow S U(3)_{c} \times S U(2)_{L} \times U(1)_{Y} \times$ $U(1)_{\tilde{I}} \rightarrow S U(3)_{c} \times S U(2)_{L} \times U(1)_{Y}$, only the minimal sets of Higgs fields are light.

To see our coupling unification strategy in this model, we first discuss the $S U(5)$ unification without supersymmetry. As is clear, in this case, we will need extra fields beyond the SM fields below the $S U(5)$ unification scale. For this purpose, we introduce $n_{3}$ real scalar $S U(2)_{L}$ triplets with $Y=0$ and $n_{8}$ real scalar color octets with $Y=0$. The coupling evolution equations in this case are the following:

$$
\begin{aligned}
& \mu \frac{d \alpha_{1}^{-1}}{d \mu}=-\frac{1}{2 \pi}\left(\frac{41}{10}\right), \\
& \mu \frac{d \alpha_{2}^{-1}}{d \mu}=\frac{1}{2 \pi}\left(\frac{19}{6}-\frac{n_{3}}{3} \theta\left(\mu-M_{3}\right)\right), \\
& \mu \frac{d \alpha_{3}^{-1}}{d \mu}=\frac{1}{2 \pi}\left(7-\frac{n_{8}}{2} \theta\left(\mu-M_{8}\right)\right),
\end{aligned}
$$

where $M_{3,8}$ stand for the masses of the triplet $(\mathbf{1}, \mathbf{3}, 1)$ and octet $(\mathbf{8}, \mathbf{1}, 0)$ fields, respectively. Solving these equations with $n_{3}=5$ with mass $M_{3}=5 \mathrm{TeV}$ and $n_{8}=3$ with mass $M_{8}=200 \mathrm{TeV}$, we find that the $S U(5)$ gauge coupling unification is achieved at $M_{U}=6.8 \times 10^{15} \mathrm{GeV}$.

Let us now proceed to $S O(10)$ unification, i.e., the running of the $g_{I}$ coupling from its breaking scale (which does not affect very much) to where it unifies with the $S U(5)$ coupling evolving after the $S U(5)$ unification scale. We see that due to the small value of $g_{I}$ required to get the relic density from the freeze-in mechanism, the $S O(10)$ gauge coupling unification in four dimensions is hard to obtain. We therefore assume that above the $S U(5)$ GUT scale, the model becomes five dimensional [33] with the 
fifth dimension compactified on $S^{1} / Z_{2}$ orbifold with a radius $R=M_{U}^{-1}$. In that case, if we assume that the gauge fields are in the bulk while all the matter and Higgs fields are on a brane at an orbifold fixed point, their Kaluza-Klein (KK) modes contribute to the running of the $S U(5)$ coupling whereas $U(1)_{\tilde{I}}$ being Abelian its coupling running does not get any extra contribution from the opening of fifth dimension. The evolution of the $S U(5)$ gauge coupling $\left(\alpha_{5}\right)$ obeys

$$
\begin{aligned}
\mu \frac{d \alpha_{5}^{-1}}{d \mu}= & \frac{1}{2 \pi}\left(\frac{43}{3}-\frac{1}{6}-\frac{5}{6}\left(1+n_{3}+n_{8}\right)\right. \\
& \left.+\frac{55}{3} \sum_{n=1} \theta\left(\mu-\sqrt{1+n^{2}} M_{U}\right)\right) .
\end{aligned}
$$

Here, in the parenthesis of the right-hand side, $43 / 3$ is the contribution from the zero-mode $S U(5)$ gauge boson and the SM fermions, $-1 / 6$ from the 5-representation Higgs field, and $-\frac{5}{6}\left(1+n_{3}+n_{8}\right)$ from one adjoint Higgs to break the $S U(5)$ symmetry and $n_{3}+n_{8}$ adjoint Higgs field into which the triplet and octet scalars are embedded, and the last term is the contribution from the $S U(5)$ gauge boson KK modes. For the KK mode mass spectrum, we have simply added the contribution from the $S U(5)$ symmetry breaking. Once the extra dimension opens, the contribution from the KK modes changes the scale dependence of the running gauge coupling from a log to a power [33]. Thus, it is possible to unify the $S U(5)$ and $U(1)_{\tilde{I}}$ couplings into $S O(10)$ coupling as desired. This is shown in Fig. 4. In the figure, the $S O(10)$ gauge coupling unification is achieved at $M_{P}$ with a unified coupling $g_{S O(10)} \simeq 0.1$. This result corresponds to an allowed parameter set, $m_{\sigma} \simeq 100 \mathrm{keV}$ and $M_{Z_{B L}}=10^{14} \mathrm{GeV}$, in the right panel of Fig. 3.

As far as proton decay is concerned, the primary mode is $p \rightarrow e^{+}+\pi^{0}$ mediated by the $S U(5)$ gauge boson. The proton decay amplitude gets contribution from all the KK excitations of the SU(5) gauge fields, and we estimate the modification of a coefficient of the four-Fermi operator to be

$$
\frac{1}{M_{U}^{2}} \rightarrow \frac{1}{M_{U}^{2}}\left(1+\sum_{n=1}^{\infty} \frac{1}{1+n^{2}}\right) \simeq \frac{2.08}{M_{U}^{2}} \equiv \frac{1}{\Lambda^{2}}
$$

Then, (ignoring threshold effects) the proton lifetime is estimated as

$$
\tau_{p} \simeq \frac{\Lambda^{4}}{\alpha_{U}^{2} m_{p}^{5}},
$$

where $m_{p}=0.938 \mathrm{GeV}$. Using $\alpha_{5}\left(M_{U}\right) \simeq 0.026$ and $M_{U} \simeq 6.8 \times 10^{15} \mathrm{GeV}$ from Fig. 4, we find that $\tau_{p} \simeq 2.1 \times 10^{34}$ years, which is consistent with the lower

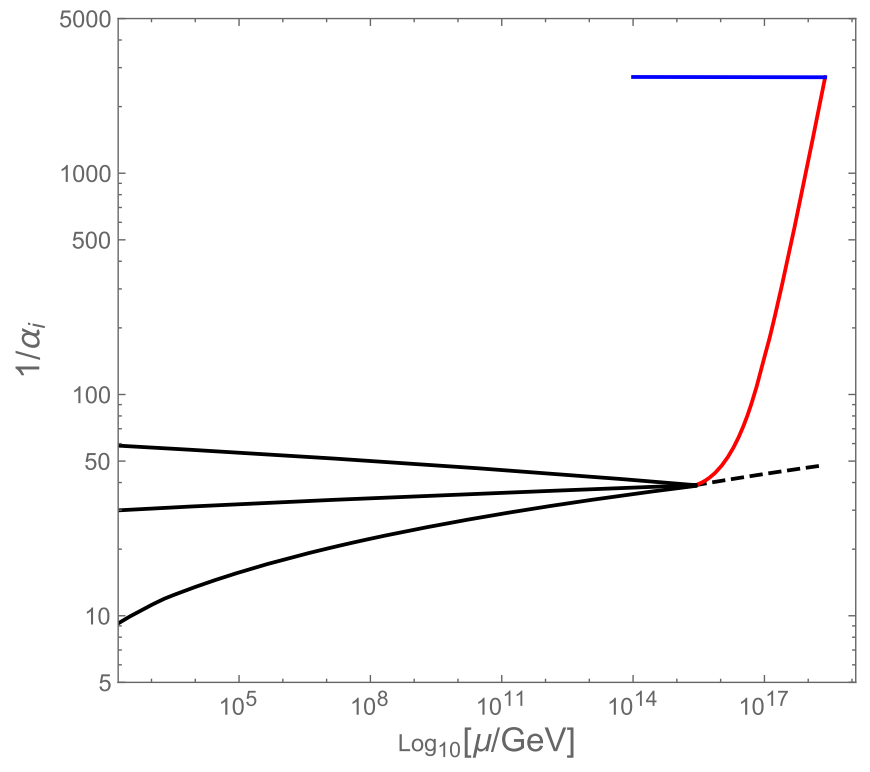

FIG. 4. Unification of gauge couplings in the presence of one extra dimension. The horizontal blue line denotes $\alpha_{\tilde{I}}^{-1}$, while solid black lines from top to bottom denote $\alpha_{1}^{-1}, \alpha_{2}^{-1}$, and $\alpha_{3}^{-1}$, respectively. Here, we have set the $U(1)_{\tilde{I}}$ gauge boson mass (corresponding to $M_{Z_{B L}}$ in the previous sections) to be $10^{14} \mathrm{GeV}$ as an example. The red curve represents the running of $\alpha_{5}^{-1}$ in the presence of the gauge boson KK modes. For a comparison with four-dimensional theory, we show the dashed line for the $S U(5)$ without the KK mode contributions.

bound $\tau_{p} \geq 1.6 \times 10^{34}$ years from the Super-Kamionkande results [34]. More importantly, we would expect that $p \rightarrow$ $e^{+} \pi^{0}$ should be observable in the next round of proton decay searches at Hyper-Kamiopkande [35] or the model will be ruled out.

\section{CONCLUDING REMARKS}

We have presented a minimal model based on a $U(1)_{B-L}$ extension of the standard model where the $B-L$ breaking Higgs field plays the role of a decaying dark matter. We discuss two regions of the DM masses: one light mass region in the $\mathrm{keV}$ to $\mathrm{MeV}$ range and another where the $\mathrm{DM}$ mass is in the PeV range. In both cases, due to the stability requirement of the dark matter, the freeze-in mechanism is required to understand the observed relic density of DM. We then discuss how the model can be tested in the FASER and other Lifetime Frontier experiments. Finally, we show how the model can emerge from an $S O(10)$ GUT model. Coupling unification in this case requires that the model be part of a five-dimensional space-time with the compactification radius being of the order of the inverse of the $S U(5)$ unification scale $M_{U}$. This embedding reflects itself in an enhanced decay rate for the proton due to extra gauge KK mode contributions, which we have estimated. The model may have $\mathrm{TeV}$ scale hypercharge neutral weak isotriplet and color octet scalars, which have interesting 
LHC phenomenology [36,37]. Discussion of this phenomenology is beyond the scope of this paper. There are also ranges for the RHN masses in the model where resonant leptogenesis can generate the baryon asymmetry of the universe. This will be the subject of a forthcoming publication.

\section{ACKNOWLEDGMENTS}

The work of R.N.M. is supported by the National Science Foundation Grants No. PHY-1620074 and No. PHY-1914631, and the work of N.O. is supported by the U.S. Department of Energy Grant No. DESC0012447.
[1] P. Minkowski, Phys. Lett. 67B, 421 (1977).

[2] R. N. Mohapatra and G. Senjanović, Phys. Rev. Lett. 44, 912 (1980).

[3] T. Yanagida, Conf. Proc. C7902131, 95 (1979).

[4] M. Gell-Mann, P. Ramond, and R. Slansky, Conf. Proc. C790927, 315 (1979).

[5] S. L. Glashow, NATO Sci. Ser. B 61, 687 (1980).

[6] R. E. Marshak and R. N. Mohapatra, Phys. Lett. 91B, 222 (1980).

[7] R. N. Mohapatra and R. E. Marshak, Phys. Rev. Lett. 44, 1316 (1980).

[8] A. Davidson, Phys. Rev. D 20, 776 (1979).

[9] L. Basso, A. Belyaev, S. Moretti, and C. H. ShepherdThemistocleous. Phys. Rev. D 80, 055030 (2009).

[10] A. A. Abdelalim, A. Hammad, and S. Khalil, Phys. Rev. D 90, 115015 (2014).

[11] S. Iso, N. Okada, and Y. Orikasa, Phys. Lett. B 676, 81 (2009); Phys. Rev. D 80, 115007 (2009).

[12] J. Heeck, Phys. Lett. B 739, 256 (2014).

[13] M. Bauer, P. Foldenauer, and J. Jaeckel, J. High Energy Phys. 07 (2018) 094.

[14] S. Blasi, V. Brdar, and K. Schmitz, arXiv:2004.02889.

[15] R. N. Mohapatra and N. Okada, arXiv:1908.11325.

[16] S. Heeba and F. Kahlhoefer, Phys. Rev. D 101, 035043 (2020).

[17] L. J. Hall, K. Jedamzik, J. March-Russell, and S. M. West, J. High Energy Phys. 03 (2010) 080.

[18] For different B-L models with right-handed neutrino as dark matter, see A. Biswas and A. Gupta, J. Cosmol. Astropart. Phys. 09 (2016) 044; 05 (2017) A01; K. Kaneta, Z. Kang, and H.-S. Lee, J. High Energy Phys. 02 (2017) 031.

[19] J. L. Feng, I. Galon, F. Kling, and S. Trojanowski, Phys. Rev. D 97, 035001 (2018).

[20] S. Bertolini, L. Di Luzio, and M. Malinsky, Phys. Rev. D 81, 035015 (2010).
[21] E. Ma, Phys. Rev. Lett. 115, 011801 (2015).

[22] L. Canetti, M. Drewes, and M. Shaposhnikov, Phys. Rev. Lett. 110, 061801 (2013).

[23] N. Aghanim et al. (Planck Collaboration), arXiv:1807 .06209 .

[24] M. G. Baring, T. Ghosh, F. S. Queiroz, and K. Sinha, Phys. Rev. D 93, 103009 (2016).

[25] S. Alekhin et al., Rep. Prog. Phys. 79, 124201 (2016).

[26] T. Akesson et al. (LDMX Collaboration), arXiv:1808 .05219 .

[27] M. J. Dolan, T. Ferber, C. Hearty, F. Kahlhoefer, and K. Schmidt-Hoberg, J. High Energy Phys. 12 (2017) 094.

[28] P. Ilten, J. Thaler, M. Williams, and W. Xue, Phys. Rev. D 92, 115017 (2015).

[29] P. Ilten, Y. Soreq, J. Thaler, M. Williams, and W. Xue, Phys. Rev. Lett. 116, 251803 (2016).

[30] M. Bauer, P. Foldenauer, and J. Jaeckel, J. High Energy Phys. 07 (2018) 094.

[31] M. Aartsen et al. (IceCube Collaboration), Phys. Rev. Lett. 111, 021103 (2013).

[32] N. Okada, S. Okada, and D. Raut, Phys. Lett. B 780, 422 (2018).

[33] K. R. Dienes, E. Dudas, and T. Gherghetta, Phys. Lett. B 436, 55 (1998); Nucl. Phys. B537, 47 (1999).

[34] K. Abe et al. (Super-Kamiokande Collaboration), Phys. Rev. D 95, 012004 (2017).

[35] K. Abe et al., arXiv:1109.3262.

[36] See for example, J. Gunion, R. Vega, and J. Wudka, Phys. Rev. D 42, 1673 (1990); H. E. Logan and M. Roy, Phys. Rev. D 82, 115011 (2010); C. Englert, E. Re, and M. Spannowsky, Phys. Rev. D 88, 035024 (2013).

[37] T. Han, I. Lewis, and Z. Liu, J. High Energy Phys. 12 (2010) 085 . 